\title{
REGLAMENTOS FUTUROS DE MÉXICO: UN PUNTO DE VISTA PERSONAL
}

\author{
Emilio Rosenblueth \\ Instituto de Ingeniería, UNAM, y \\ Centro de Investigación Sísmica
}

\begin{abstract}
RESUMEN
Esta nota se refiere a las disposiciones reglamentarias para diseño sísmico en la República Mexicana. Se arguye que tiene mayor importancia asegurarse de que un reglamento se cumple que mejorarlo. Se abordan principalmente aspectos de optimación, zonación, microzonación y el método de espectros de diseño.
\end{abstract}

\section{ABSTRACT}

This note concerns earthquake resistant provisions for Mexico. It is argued that compliance with a code is more importint than its improvement. The main facets addressed are optimization, zonation, microzonation, and the design spectrum method.

\section{INTRODUCCIÓN}

No hay organismo oficial que haya dado a conocer planes para reglamentos futuros. Lil presente nota contiene opiniones del autor mezcladas con algunos buenos deseos. Se refiere a disposiciones reglamentarias para diseño sísmico de estructuras en la República Mexicana incluyendo el Distrito Federal.

* Triducción de "Future Mexican codes: a personal vicw", que el autor presentó en cl Works/ıop on Influencing the Next Generation of Seismic Design Practices, verificado en Ixtapa, Gro el 29 de septiembre 1992 con el patrocinio de la Structural Enginecrs Associa- tion of California (SEAOC) y la United States Federal Emergency Management Agency (FEMA). 
E. ROSENBLUETH

Tiene mayor importancia el que un reglamento existente se cumpla a que se le mejore. Las cláusulas más cruciales de un reglamento son aquellas que prácticamente aseguren su observancia. Dicho esto, sin embargo, me referiré principalmente a cómo mejorar los reglamentos. Los temas centrales que abordaré se refieren a optimación, zonación, microzonación y el método de espectros de diseño.

\section{OPTIMACIÓN DE REGLAMENTOS}

En primera instancia la mayoría de nosotros sostendríamos que tratamos de hacer todo lo mejor posible. En otras palabras, procuramos optimar. No obstante, seguimos tomando multitud de decisiones a sentimiento. ¿De qué otra manera fijamos factores de importancia, coeficientes de reducción de resistencia, factores $Q$, requisitos de regularidad, en gran medida coeficientes de cortante basal o periodos de retorno con fines de diseño y la necesidad de realizar análisis dinámicos o cuándo permitir métodos simplificados en ciertos reglamentos, eximir algunas construcciones del requisito de diseño sísmico y cuánto relajar los requisitos para estructuras existentes con respecto a las nuevas?

Si nuestra labor consistiera en diseñar una estructura individual carente de importancia, sin tener que apegarnos a restricciones normativas, estaría justificado que prefiriéramos la intuición -- llámese criterio ingenieril -- al análisis cuantitativo para establecer estas cantidades. ¡Pero los reglamentos afeclan miles de estructuras!

El siguiente ejemplo (Hoffman, Slovic y Rorer, 1968) es elocuente en cuanto a por qué no hemos de confiar en la intuición. A cinco radiólogos independientes se les dio información acerca de 96 casos de úlceras gástricas sospechosas y se les pidió que evaluaran cada uno desde el punto de vista oncológico. A la semana se les presentó la misma información en otro orden. Las correlaciones entre los primeros y segundos diagnósticos individuales varió entre 0.60 y 0.92 . Schoemaker y Russo (1992) comentan irónicamente: " Este ejemplo... apoya el consejo estándar de buscar una segunda opinión cuando se trata de decisiones médicas importantes. ¡Lo que sorprende es que pueda obtenerse la segunda opinión del mismo médico!" Los mismos autores citan correlaciones que típicamente van de 0.2 a 0.3 entre datos reales y su estimación intuitiva en diversas disciplinas, frecuentemente cuando son especialistas quienes estiman.

¿Califica mejor nuestro criterio en ingeniería sismica? La verdad es que los médicos son más precisos cuando predicen el tiempo de vida que le queda a un paciente que cuando pretendemos 
REGLAMENTOS FUTUROS DE MEXICO

predecir el instante en que ocurrirá el siguiente macrosismo, no se diga en lo que atañe a sus características detalladas.

Hasta hace pocos años era impensable la optimación cuantitativa de un reglamento. No se podía hablar de cuánto debía invertir la sociedad para salvar una vida humana; sin embargo, el propósito de los reglamentos era proteger las vidas e integridad física de las personas, no interesaba el costo que implicaba cumplir con el propósito explícito ni a cuánto ascenderían las pérdidas económicas directas e indirectas. Hoy existe preocupación generalizada con estos conceptos y cierto grado de consenso en cuanto a que, en los Estados Unidos, la sociedad debe invertir entre uno y dos millones de dólares por vida humana que se salve durante un temblor. (Tal vez haya quienes se inclinen por el intervalo de $\$ 200000$ a $\$ 5000000$ para salvar una vida anónima en general pero las cifras dependen de la causa potencial de fallecimiento.)

Fan México, con base en el Producto Interno Bruto per cípita, serían más apropiadas cifras del orden de un décimo de las anteriores. Aun si estuvieran seriamente erradas estas cantidades, se afectarian poco las decisiones óptimas en ingeniería sísmica. Por ejemplo, varias estimaciones colocan las pérdidas econónicas totales debidas al temblor de 1985 aproximadamente en 14000 millones de dólares. Posiblemente se perdieron 10000 vidas.

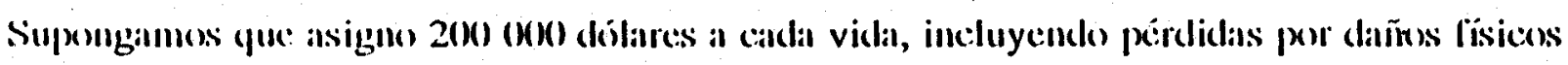
a personas y concluyo que cierto tipo de estructura debe diseñarse óptimamente para un coeficiente de corte basal de $\mathbf{0 . 2 0}$. Ahora me convencen ustedes que debo duplicar la cifra de $\$ 200$ 000. Casi cualquier modelo razonable de ocurrencia de macrosismos llevará a aumentar el coeficiente de 0.20 a 0.21 aproximadamente.

Los efectos de errores de este orden son aún menores en los Estados Unidos, donde los desastres naturales sistemáticamente causan dramáticamente menos víctimas y mayores pérdidas económicas.

Adicionalmente es mucho lo que podemos lograr pensando en términos de optimación sin comprometernos al valor social de una vida. Por tanto, si admitimos que el factor de importancia reglamentario de 1.5 es óptimo para el Distrito Federal, deberá ser (pero no lo es) apreciablemente menor en Acapulco ya que en el área epicentral los parámetros relevantes del movimiento del terreno se saturan para magnitudes elevadas; por ejemplo, debido a la gran extensión del área de ruptura que causa a un temblor de magnitud elevada, los deslizamientos en 
E. ROSENBLUETH

sitios lejanos contribuyen significativamente a la energía liberada durante el fenómeno pero no a la aceleración máxima del terreno directamente arriba del área de ruptura. Como veremos hay también implicaciones en lo que toca a zonación.

Una implicación de gran alcance resulta de que, si un parámetro de diseño no difiere demasiado de su valor óptimo, la optimación sólo producirá, en la utilidad, incrementos de segundo orden. Se sigue que al redactar un reglamento deberíamos concentrarnos en unos cuantos conceptos para los que podemos estar muy lejos del óptimo, como la evaluación de la sismicidad, los efectos de sitio y las consecuencias del comportamiento no lineal en sistemas con varios grados de libertad, en preferencia a insistir en nuestros refinamientos predilectos, que pueden complicar el documento, con lo que deja de ser atractivo y digno de crédito. $Y$ en investigación deberíamos adoptar los mismos criterios y además prestar mayor atención a las innovaciones.

\section{ZONACIÓN}

Es usual que un documento oficial introduzea un parámetro del movimiento del terreno, tal como la aceleración máxima de éste o su aceleración efectiva máxima, que supuestamente es la cantidad relevante para el diseño de toda estructura; presente mapas con curvas de valores constantes de ese parámetro en roca o en terreno firme para ciertos periodos de recurrencia, y ya sea que se exija que toda estructura se diseñe para el valor del parámetro relevante que, en el mapa cuyo periodo de recurrencia se asocia a la importancia de la estructura, corresponde al sitio donde se erigirá ésta (como sucede en Costa Rica) o bien defina zonas y especifique que toda estructura en una zona dada se diseñe para el máximo valor del parámetro que se halla en esa zona para un periodo de recurrencia dado. Las fronteras entre las zonas suelen coincidir con valores constantes que tiene el parámetro relevante en uno de los mapas.

Nada de esto puede estar bien. Diversos lipos de estruclura, digamos con distintos periodos fundamentales de vibación, se ven afectados preponderantemente por diferentes parámetros del movimiento del terreno, los que no necesariamente son proporcionales entre sí. Una norma propuesta en México (Manual de Obras Civiles, 1992) da atención a este hecho, pero no la suficiente.

Para ilustrar el punto movámonos a lo largo de una línea apartándonos de la costa del Pacífico (donde se originan nuestros mayores eventos) y acercándonos al Eje Neovolcánico (rico 
en temblores locales). En cierto intervalo encontraremos que la demanda de cortante basal disminuye para las estructuras flexibles a la vez que aumenta para las rígidas. Por otra parte es bastante arbitraria la elección del periodo de recurrencia. Además, debemos zonificar -en otra forma resulta excesiva la ambigüedad en los mapas de dimensiones manejables- por la misma razón, así como para facilitar la aplicación de las normas, las fronteras entre zonas deberían coincidir con límites jurisdiccionales.

Por último, si diseñamos para el valor máximo de una zona estamos siendo conservadores, en ocasiones de manera excesiva; el óptimo suele yacer más cerca del promedio que del máximo.

En ciertas circunstancias el problema de optimar la zonación puede reducirse a una sola dimensión. Si entonces adoptamos el criterio de diseñar para el valor máximo que se presenta en la zona, podemos acudir a un método sencillo desarrollado para la optimación de catálogos (Lind, Rukos y Barrera, 1976). También en una dimensión, si sustituimos el criterio de diseño óptimo por el de diseño para el valor máximo, podemos aplicar un principio desarrollado por Lind (1975). En la UNAM se están desarrollando métodos eficientes para problemas que no se pueden reducir a una sola dimensión.

Pueden aplicarse comentarios semejantes a los criterios en cuanto a la zonación que se relaciona con susceptibilidad a deslizamientos de tierra o roca y a licuación.

\section{MICROZONACIÓN}

Mucho de lo que he expresado respecto a zonación es también válido en microzonación. Dos diferencias obvias consisten en que: 1) Las calles pueden constituir fronteras entre microzonas, y 2) Son decisivos los accidentes topográficos y las condiciones locales del suelo.

Esta última consideración nos lleva al problema de la caracterización del sitio. Donde la roca yace a profundidad pequeña o moderada y el contraste de impedancias es grande, el periodo dominante del terreno está bien definido. No hay razón para ignorar este útil parámetro en todas partes por el sólo hecho de que su valor sea vago en otros lugares; incluso, en los primeros sitios a que me referí, bien puede autorizarse la determinación local de este parámetro con base en registros de microtremores. Los sitios de otra naturaleza merecen atención adicional y la están recibiendo. En general la caracterización del suelo debe comprender no sólo la estratigrafía, el espesor y la rigidez o densidad de cada material sino también su índice de plasticidad para tener 


\section{E. ROSENBLUETH}

una buena idea de la dependencia del módulo de rigidez secante y del amortiguamiento con respecto a la deformación (Romo, 1990).

Vale la pena explorar si podemos describir adecuadamente los espectros mediante 4-6 parámetros en vez de hacerlo mediante las ordenadas espectrales en un gran número de periodos. (Joyner y Boore trabajan actualmente en este enfoque. Hay un antecedente en el trabajo de Newmark con aceleración, velocidad y desplazamiento máximos del terreno.) También debe incluirse en la descripción la duración del movimiento intenso.

\section{ESPECTROS DE DISEÑO}

A pesar de múltiples objeciones me temo que tendremos que seguir usando durante muchos años los espectros de diseño, al menos como una alternativa en los reglamentos. Deberíamos mejorar la manera como obtenemos dichos espectros y lo que hacemos con ellos, por ejemplo teniendo en cuenta la acción simultánea de los diversos componentes del movimiento del terreno.

Primero, parece poco satisfactorio derivar una familia de espectros de respuesta a partir de un acelerograma único, sea que hagamos aleatorias las fases de sus ondas senoidales constitutivas o que acudamos a Métodos ARMA (Samaras y col, 1987). Nos interesan los espectros asociados al universo de temblores que pueden generarse en todas" las fuentes potenciales, con parámetros focales aleatorios (Hartzell, 1978; Ordaz y col, 1989) y el empleo de funciones de Green empíricas (Irikura, 1983; Izutani y Katagari, 1992). Una vez generados los movimientos simulados podemos calcular los tamaños y formas de los espectros de respuesta lineal, así como las duraciones de movimientos intensos en los sitios de interés y tal vez, como se sugiere en Microzonación, describir estos espectros mediante un número pequeño de parámetros.

Nuestros reglamentos actuales modifican intuitivamente los espectros calculados para tener en cuenta incertidumbres en el movimiento del terreno y en el comportamiento estructural, así como ciertas consideraciones de diseño óptimo (como la disminución de las ordenadas espectrales con un exponente menor del periodo que lo que pudiera inferirse de las esperanzas de ordenadas espectrales) pero sería deseable llegar a estas modificaciones mediante análisis cuantitativos.

Existen métodos razonablemente bien establecidos de análisis estructural dentro del rango lineal que parten de espectros de respuesta. El método CQC extendido y el empleo de espectros- 
equivalentes (Berrah y Kausel,1992) lidian incluso con estructuras cuyos apoyos se mueven asíncronamente. Mas necesitamos métodos prácticos, aproximados para aprovechar los espectros de comportamiento lineal en el tratamiento de la interacción suelo-estructura (véase Esteva, 1992 en lo que respecta a las incertidumbres que aquejan a este problema), daño acumulado, uso de métodos energéticos y la estimación de desplazamientos para poder aplicar enfoques tan atractivos como el método de Moehle (1992) de diseño por desplazamientos.

\section{COMENTARIOS FINALES}

Es concebible que en pocos años las disposiciones teglamentarias mexicanas:

- Incluyan cláusulas que virtualmente aseguren el cumplimiento con tales disposiciones;

- Procuren producir diseños óptimos con base en análisis cuantitativos, por lo menos en lo concerniente a zonación, microzonación, cálculo de espectros de respuesta y de diseño y su aplicación fuera del intervalo de comportamiento lineal, considerando explícitamente conceptos tales como la acumulación de daño, los coeficientes de importancia, los factores de carga, los coeficientes reductores de resistencia, los factores $\mathrm{Q}, \operatorname{los}$ requisitos de regularidad, periodos de recurrencia, tipo de análisis requerido y provisiones especiales para las estructuras existentes; $y$

- Se tornen más sencillas, más dignas de crédito y más atractivas, aun sacrificando algo la . precisión.

Espero que la investigación dirigida a mejorar nuestros reglamentos en asuntos de índole cuantitativo se concentre en temas sobre los que todavía podemos estar muy lejos del óptimo y que se ejerza mayor esfuerzo en las innovaciones, olvidándonos de tantos refinamientos.

\section{Reconocimiento}

Agradezco a Mario Ordaz su valiosa ayuda en la preparación de esta nota. 


\section{REFERENCIAS}

Berrah, M y Kausel, E, 1992, "Response spectrum analysis of structures subjected to spatially varying motions", Earthquake Engineering and Structural Dynamics, 24, 427-70.

Esteva, L, 1992, "Nonlinear seismic response of soft-first-story buildings subjected to narrow band accelerograms", Earthquake Spectra, 8, 3, 373-89.

Hartzell, S M, 1978, "Earthquake aftershocks as Green's functions", Geophys Res Letters, 5, 1-4.

Hoffman, P J, Slovic, P y Rorer, L G, 1968, "An analysis-of-variance model for assessment of configural cue utilization in clinical judgment", Psychological Bulletin, 69, 338-49, citado en Schoemaker and Russo (1992).

Irikura, K, 1983, "Semi-empirical estimation of strong ground motions during large earthquakes", Bull Disas Prev Res Inst, 33, 63-104.

Izutani, Y y Katagiri, F, 1992, "Empirical Green's function corrected for source effect", Earthquake Engineering and Structural Dynamics, 21, 341-49.

Lind, N C, 1975, "Optimization of a discrete solution set", Univ of Waterloo, Solid Mechanics Division Report.

Lind, N C, Rukos, E y Barrera, R, 1976, "Optimación de catálogos de productos estandarizados", Revista Ingenieria, Jun, 129-36.

Manual de Obras Civiles, Parte III, 1992, Comisión Federal de Electricidad, México, en prensa.

Moehle, J P, 1992, "Displacement based design of RC structures subjected to earthquakes", Earthquake Spectra, 8, 3, 403-28.

Ordaz, M, Jara, J M y Singh, S K, 1989, "Riesgo sísmico y espectros de diseño para el estado de Guerrero", Informe Interno, Instituto de Ingeniería, UNAM, México.

Romo, M P, 1990, "Comportamiento dinámico de la arcilla de la ciudad de México y sus repercusiones en la ingeniería de cimentaciones", Memorias del Simposio El Subsuelo de la Cuenca del Valle de México, Soc. Mexicana de Mecánica de Suelos, 83-94.

Samaras, E, Shinozuka, M y Tsutui, A, 1987, "Time series generation using the Auto-Regressive-Moving-Average Model", Stochastic Mechanics, Columbia University, New York, ed: M Shinozuka.

Schoemaker, P J H y Russo, J E, 1992, A pyramid of decision approaches, Graduate School of Business, The University of Chicago, Chicago, $\mathrm{i}+28 \mathrm{pp}$. 